\title{
Around a problem of Nicole Brillouët-Belluot
}

\author{
JANUSZ MORAWIEC
}

Abstract. We determine nontrivial intervals $I \subset(0,+\infty)$, numbers $\alpha \in \mathbb{R}$ and continuous bijections $f: I \rightarrow I$ such that $f(x) f^{-1}(x)=x^{\alpha}$ for every $x \in I$.

Mathematics Subject Classification (2010). Primary 39B22; Secondary 39B12, 26A18.

Keywords. Iterative functional equation; continuous bijection; iterate, monotonic solution.

\section{Introduction}

In [7] (see also [6, page 358], [1]) the authors, motivated by a freshman mistake when for a student the inverse $f^{-1}$ and the reciprocal $1 / f$ of a given bijection $f: I \rightarrow I$ have the same meaning, come to the equation

$$
f^{-1}(x)=\frac{1}{f(x)} \quad \text { for every } x \in I,
$$

where $I \subset \mathbb{R}$. In [7] it is demonstrated that in the case where $I=(0,+\infty)$ bijections satisfying Eq. (1.1) do exist, but each such bijection must have an infinite number of discontinuities. A complete description of real bijective solutions of Eq. (1.1) was obtained in [5], along with examples illustrating the possible behavior of such solutions. In contrast to the real case there exist analytic bijections satisfying (1.1) defined on a region $I$ of the complex plane (see [5]). Equation (1.1) is a particular case of the equation $f^{m}(x)=[f(x)]^{n}$ discussed, among others, in [4] and in [13] for some integers $m$ and $n$. It was noticed in [11] (see also [9, page 293]) that solutions of equation (1.1) can be used for the construction of iterative roots of the identity. More results on iterative roots of the identity can be found, among others, in [9, Chapter XV], [10, Chapter 11].

All continuous bijective solutions $f: I \rightarrow I$ of the equation

$$
f(x) f^{-1}(x)=x^{2} \quad \text { for every } x \in I,
$$


where $I \subset \mathbb{R}$ is an interval, were determined in [12], when solving a problem posed by Nicole Brillouët-Belluot (see [3]). Equation (1.2) without the continuity assumptions on its bijective solutions were studied in [8], where a complete description of general bijective solutions of (1.2) can be found, as well as examples of discontinuous bijections satisfying (1.2). Equation (1.2) can be written in the form

$$
f^{2}(x) x=[f(x)]^{2} \quad \text { for every } x \in I,
$$

however, now we do not need to assume that $f$ is a bijection. It turns out that in the case where $I=(0,+\infty)$ Eqs. (1.2) and (1.3) have the same continuous solutions (see [2]).

In this paper we are interested in continuous bijections $f: I \rightarrow I$ such that

$$
f(x) f^{-1}(x)=x^{\alpha} \quad \text { for every } x \in I,
$$

where $\alpha \in \mathbb{R}$ and $I \subset \mathbb{R}$ is a nontrivial interval. Clearly, Eq. (E) generalizes both Eqs. (1.1) and (1.2).

\section{Preliminary}

For the convenience of the reader, we begin with repeating results on continuous bijective solutions of Eqs. (1.1) and (1.2) defined on a real interval.

Theorem 2.1 (see [5]). There is no continuous bijective solution of equation (1.1) defined on a nontrivial interval of the real line.

Theorem 2.2 (see [12]). Assume that $I \subset \mathbb{R}$ is a nontrivial interval. If $f: I \rightarrow I$ is a continuous bijective solutions of equation (1.2), then one of the following assertions hold:

(i) $I$ is bounded or $0 \notin \mathrm{cl} I$ and $f(x)=x$ on $I$;

(ii) $I \neq \mathbb{R}$ is unbounded with $0 \in \operatorname{cl} I$ and there exists $c>0$ such that $f(x)=$ $c x$ on the half-line $J \subset$ I ending with 0 and $f(x)=x$ on $I \backslash J$;

(iii) $I$ is bounded with $\mathrm{cl} I=-\operatorname{cl} I$ and $f(x)=-x$ on $I$;

(iv) $I=\mathbb{R}$ and either there exists $a<0$ such that $f(x)=$ ax on $I$ or there exist $b, c>0$ such that $f(x)=b x$ on $(-\infty, 0]$ and $f(x)=c x$ on $[0,+\infty)$.

From now on we assume that $\alpha \in \mathbb{R} \backslash\{0,2\}$ and $I \subset \mathbb{R}$ is a nontrivial interval.

Following the idea from [12] we begin with finding useful formulas for iterates of bijective solutions $f: I \rightarrow I$ of equation (E). For this purpose, we define a sequence $\left(a_{n}\right)_{n \in \mathbb{Z}}$ putting

$$
a_{0}=1, \quad a_{1}=\alpha \quad \text { and } \quad a_{n+1}=\alpha a_{n}-a_{n-1} \quad \text { for every } n \in \mathbb{N} \text {. }
$$


Lemma 2.3. Assume that $f: I \rightarrow I$ is a bijection satisfying (E). Then for every $n \in \mathbb{N}$ we have:

$$
\begin{aligned}
& f^{n+1}(x)=\frac{[f(x)]^{a_{n}}}{x^{a_{n-1}}} \quad \text { for every } x \in I \backslash\{0\} ; \\
& f^{-n}(x)=\frac{x^{a_{n}}}{[f(x)]^{a_{n-1}}} \quad \text { for everyx } \in I \backslash\{0\} .
\end{aligned}
$$

Proof. Putting $f(x)$ instead of $x$ in (E) we get $f^{2}(x)=\frac{[f(x)]^{\alpha}}{x}=\frac{[f(x)]^{a_{1}}}{x^{a_{0}}}$ for every $x \in I \backslash\{0\}$. Hence (2.2) holds for $n=1$. Fix $n \in \mathbb{N}$ and assume that (2.2) holds. Then $f^{n+2}(x)=f^{n+1}(f(x))=\frac{\left[f^{2}(x)\right]^{a_{n}}}{[f(x)]^{a_{n-1}}}=\frac{[f(x)]^{\alpha a_{n}}}{x^{a_{n}}[f(x)]^{a_{n-1}}}=\frac{[f(x)]^{a_{n}+1}}{x^{a_{n}}}$ for every $x \in I \backslash\{0\}$.

Since $f$ satisfies (E), so does $f^{-1}$. Therefore (2.2) and (E) yield $f^{-(n+1)}(x)=$ $\frac{\left[f^{-1}(x)\right]^{a_{n}}}{x^{a_{n}-1}}$ for all $n \in \mathbb{N}$ and $x \in I \backslash\{0\}$. Replacing $x$ by $f(x)$ we obtain (2.3).

We finish this section with a lemma which will be useful for determining decreasing bijective solutions of equation (E).

Lemma 2.4. Assume that $f: I \rightarrow I$ is a decreasing bijection satisfying $(E)$. Then the function $g: I \rightarrow I$ given by $g(x)=f^{2}(x)$ is an increasing bijection and

$$
g(x) g^{-1}(x)=x^{\alpha^{2}-2} \quad \text { for every } x \in I .
$$

Proof. It is clear that the function $g$ is an increasing bijection. By Lemma 2.3 we have $g(x) g^{-1}(x)=f^{2}(x) f^{-2}(x)=\frac{[f(x)]^{a_{1}}}{x^{a_{0}}} \cdot \frac{x^{a_{2}}}{[f(x)]^{a_{1}}}=x^{a_{2}-a_{0}}=x^{\alpha^{2}-2}$ for every $x \in I \backslash\{0\}$. If $0 \in I$, then $g(0)=f^{2}(0)=0$.

\section{Main results}

From now on we assume that $\operatorname{cl} I \subset[0,+\infty)$. However, all presented results can be extended to the general case $I \subset \mathbb{R}$ according to the following observation.

Remark 3.1. Assume that $f: I \rightarrow I$ is a bijection satisfying (E). Then the function $h:-I \rightarrow-I$ given by $h(x)=-f(-x)$ is a bijection and $h(x) h^{-1}(x)=$ $(-x)^{\alpha}$ for every $x \in-I$.

Necessary conditions on $\alpha$ and $I$ for equation (E) to have a continuous bijective solution $f: I \rightarrow I$ gives the following lemma.

Lemma 3.2. Assume that $f: I \rightarrow I$ is a bijection satisfying (E).

(i) If $f$ is increasing, then $\alpha>0$ and either $\operatorname{cl} I=[0,1]$ or $\operatorname{cl} I=[1,+\infty)$ or $\mathrm{cl} I=[0,+\infty)$.

(ii) If $f$ is decreasing, then either $\alpha<0$ and $I=(0,+\infty)$ or $\alpha=-2$ and there exists $a \in(0,1)$ such that $\operatorname{cl} I=\left[a, a^{-1}\right]$. 
Proof. Put $a=\inf I, b=\sup I$.

(i) Clearly, $a=\lim _{x \rightarrow a^{+}} f(x)=\lim _{x \rightarrow a^{+}} f^{-1}(x)$ and $b=\lim _{x \rightarrow b^{-}} f(x)=$ $\lim _{x \rightarrow b^{-}} f^{-1}(x)$.

If $a>0$, then (E) implies $a^{2}=a^{\alpha}$, and since $\alpha \neq 2$, we have $a=1$. Similarly, if $b<+\infty$, then $b=1$.

Since $f$ is increasing, so is the function

$$
I \ni x \longmapsto f(x) f^{-1}(x) \in I \cdot I .
$$

Thus $\alpha>0$.

(ii) Clearly, $a=\lim _{x \rightarrow b^{-}} f(x)=\lim _{x \rightarrow b^{-}} f^{-1}(x)$ and $b=\lim _{x \rightarrow a^{+}} f(x)=$ $\lim _{x \rightarrow a^{+}} f^{-1}(x)$. If $a>0$, then (E) implies $a^{2}=b^{\alpha}$. Hence $b<+\infty$ and $b^{2}=a^{\alpha}$, by (E). Consequently,

$$
\left(\frac{a}{b}\right)^{\alpha+2}=\frac{a^{2}}{b^{\alpha}} \cdot \frac{a^{\alpha}}{b^{2}}=1 .
$$

Since $a<b$, we have $\alpha=-2$, and hence $b=a^{-1}$. Similarly, if $b<+\infty$, then $\alpha=-2$ and $a=b^{-1}$.

Since $f$ is decreasing, so is the function given by (3.1). Thus $\alpha<0$.

The next lemma gives useful information about the behavior of the sequence $\left(a_{n}\right)_{n \in \mathbb{Z}}$ defined by $(2.1)$.

Lemma 3.3. Assume that $\alpha>0$. If

$$
a_{n-1}<a_{n+1} \text { for every } n \in \mathbb{N} \text {, }
$$

then $\alpha>2, \lim _{n \rightarrow \infty} \frac{a_{n}}{a_{n-1}}=\beta>1$ and

$$
\beta+\frac{1}{\beta}=\alpha .
$$

In particular, $\lim _{n \rightarrow \infty} a_{n}=+\infty$.

Proof. From (3.2) and (2.1) we obtain that $2 a_{n-1}<\alpha a_{n}$ for every $n \in \mathbb{N}$. Now, by induction we see that $a_{n-1}>0$ for every $n \in \mathbb{N}$.

Define a sequence $\left(b_{n}\right)_{n \in \mathbb{N}}$ putting $b_{n}=\frac{a_{n}}{a_{n-1}}$. Obviously, $b_{n}>0$ for every $n \in \mathbb{N}$. Moreover, $b_{2}=\frac{\alpha^{2}-1}{\alpha}<\alpha=b_{1}$. Fix $n \in \mathbb{N}$ and assume that $b_{n+1}<$ $b_{n}$. Then $b_{n+2}=\frac{\alpha a_{n+1}-a_{n}}{a_{n+1}}=\alpha-\frac{1}{b_{n+1}}<\alpha-\frac{1}{b_{n}}=b_{n+1}$. Consequently, the sequence $\left(b_{n}\right)_{n \in \mathbb{N}}$ converges. Let $\beta=\lim _{n \rightarrow \infty} b_{n}$. According to (2.1) we obtain (3.3). Hence $\alpha>2$ since $\alpha \neq 2$. By (3.2) we have $1 \leq \lim _{n \rightarrow \infty} \frac{a_{n+1}}{a_{n-1}}=$ $\lim _{n \rightarrow \infty} \frac{a_{n+1}}{a_{n}} \lim _{n \rightarrow \infty} \frac{a_{n}}{a_{n-1}}=\beta^{2}$. Using (3.3) and the fact that $\alpha \neq 2$ we get $\beta>1$. In consequence, $\lim _{n \rightarrow \infty} a_{n}=+\infty$. 
Now we are ready to determine continuous bijections satisfying (E). We begin with increasing bijections. By assertion (i) of Lemma 3.2 we have three cases to consider: $\operatorname{cl} I=[0,1], \operatorname{cl} I=[1,+\infty), \operatorname{cl} I=[0,+\infty)$.

Theorem 3.4. Assume that either $\mathrm{cl} I=[0,1]$ or $\mathrm{cl} I=[1,+\infty)$ and let $f: I \rightarrow I$ be an increasing bijection satisfying (E). Then $\alpha>2$ and

$$
f(x)=x^{\beta} \quad \text { for every } x \in I
$$

with $\beta$ satisfying (3.3).

Proof. By assertion (i) of Lemma 3.2 we have $\alpha>0$.

We first consider the case $\operatorname{cl} I=[0,1]$.

It is easy to see that every increasing bijective solution of equation (E) on $I$ can be uniquely extended to an increasing bijective solution of equation (E) on clI. Thus we may (and do) assume that $I=[0,1]$. Clearly, $f(x)=x$ if and only if $x \in\{0,1\}$.

Since all the iterates of $f$ are increasing, we have $\frac{[f(x)]^{a_{n}}}{x^{a_{n}-1}}<1$ and $\frac{x^{a_{n+1}}}{[f(x)]^{a_{n}}}<$ 1 for all $x \in(0,1)$ and $n \in \mathbb{N}$, by Lemma 2.3. Thus $x^{a_{n+1}}<[f(x)]^{a_{n}}<x^{a_{n-1}}$ for all $x \in(0,1)$ and $n \in \mathbb{N}$. In particular, (3.2) holds. Therefore, Lemma 3.3 yields $\alpha>2$.

Without loss of generality we can assume that $f(x)<x$ for every $x \in(0,1)$. Hence, by induction, we have $x<f^{-n}(x)<1$ for all $x \in(0,1)$ and $n \in \mathbb{N}$. This jointly with (2.3) and Lemma 3.3 implies

$$
1=\lim _{n \rightarrow \infty} x^{\frac{1}{a_{n-1}}} \leq \lim _{n \rightarrow \infty}\left[f^{-n}(x)\right]^{\frac{1}{a_{n-1}}}=\lim _{n \rightarrow \infty} \frac{x^{\frac{a_{n}}{a_{n-1}}}}{f(x)}=\frac{x^{\beta}}{f(x)} \leq 1
$$

for every $x \in(0,1)$ with $\beta$ satisfying (3.3).

Now we consider the case $\operatorname{cl} I=[1,+\infty)$.

Put $J=\left\{x \in \mathbb{R}: x^{-1} \in I\right\}$. We have $\operatorname{cl} J=[0,1]$. Observe that the formula $g(x)=\left[f\left(x^{-1}\right)\right]^{-1}$ defines an increasing bijection from $J$ to $J$. Moreover, $g(x) g^{-1}(x)=\left[f\left(x^{-1}\right) f^{-1}\left(x^{-1}\right)\right]^{-1}=x^{\alpha}$ for every $x \in J$. According to the previous case we conclude that $g(x)=x^{\beta}$ for every $x \in J$ with $\beta$ satisfying (3.3). Consequently, $f(x)=\left[g\left(x^{-1}\right)\right]^{-1}=x^{\beta}$ for every $x \in I$.

As an immediate consequence of Theorem 3.4 we obtain the following corollary.

Corollary 3.5. Assume that $\mathrm{cl} I=[0,+\infty)$ and let $f: I \rightarrow I$ be an increasing bijection satisfying $(E)$ such that $f(1)=1$. Then $\alpha>2$ and

$$
f(x)= \begin{cases}x^{\beta}, & \text { if } x \leq 1, \\ x^{\gamma}, & \text { if } x>1\end{cases}
$$

where $\beta$ satisfies (3.3) and $\gamma$ is such that

$$
\gamma+\frac{1}{\gamma}=\alpha
$$


Now we determine decreasing bijective solutions of (E). By assertion (ii) of Lemma 3.2 we have two cases to consider: $I=(0,+\infty), \operatorname{cl} I=\left[a, a^{-1}\right]$ with $a \in(0,1)$.

Theorem 3.6. Let $f:(0,+\infty) \rightarrow(0,+\infty)$ be a decreasing bijection satisfying (E). Then $\alpha \leq-2$ and $f$ has form (3.5) with $\beta$ satisfying (3.3) and $\gamma$ satisfying $(3.6)$.

Proof. By Lemma 2.4 the function $g=f^{2}$ is an increasing bijection satisfying (2.4). Corollary 3.5 and Theorem 2.2 imply $\alpha^{2}-2 \geq 2$. Thus assertion (ii) of Lemma 3.2 yields $\alpha \leq-2$.

We first consider the case $\alpha<-2$, that is $\alpha^{2}-2>2$.

Since $f$ has exactly one fixed point which must be equal to 1 by (E), we have $g(1)=1$. Then applying Lemma 2.3 and Corollary 3.5 we obtain

$$
\frac{[f(x)]^{\alpha}}{x}=f^{2}(x)=g(x)=\left\{\begin{array}{lll}
x^{\mu}, & \text { if } & x \leq 1, \\
x^{\nu}, & \text { if } & x>1,
\end{array}\right.
$$

with $\mu$ and $\nu$ such that

$$
\mu+\frac{1}{\mu}=\nu+\frac{1}{\nu}=\alpha^{2}-2 .
$$

In consequence $f$ has form (3.5) with $\beta=\frac{\mu+1}{\alpha}$ and $\gamma=\frac{\nu+1}{\alpha}$. Moreover, (3.3) and (3.6) hold according to (3.7).

Now we consider the case $\alpha=-2$, that is $\alpha^{2}-2=2$

As a consequence of assertion (ii) of Theorem 2.2, there exists $c>0$ such that $g(x)=c x$ for every $x \in I$. This jointly with Lemma 2.3 gives $c x=g(x)=$ $f^{2}(x)=\frac{[f(x)]^{-2}}{x}$, and hence $f(x)=\frac{1}{\sqrt{c}} x^{-1}$ for every $x \in I$. Since $f$ solves $(\mathrm{E})$, we conclude that $c=1$. Thus $f$ has form (3.5) with $\beta=\gamma=-1$.

Theorem 3.7. Assume that $\alpha=-2$ and $\operatorname{cl} I=\left[a, a^{-1}\right]$ with some $a \in(0,1)$ and let $f: I \rightarrow I$ be a decreasing bijection satisfying $(E)$. Then $f(x)=x^{-1}$ for every $x \in I$.

Proof. By Lemma 2.4 the function $g=f^{2}$ is an increasing bijection satisfying (2.4). Then applying Lemma 2.3 and assertion (i) of Theorem 2.2 we obtain $\frac{[f(x)]^{-2}}{x}=g(x)=x$ for every $x \in I$. Consequently, $f(x)=x^{-1}$ for every $x \in I$.

We end this paper with a problem.

Problem 3.8. Is the assumption $f(1)=1$ needed in Corollary 3.5?

\section{Acknowledgements}

This research was supported by Silesian University Mathematics Department (Iterative Functional Equations and Real Analysis program). 
Open Access. This article is distributed under the terms of the Creative Commons Attribution License which permits any use, distribution, and reproduction in any medium, provided the original author(s) and the source are credited.

\section{References}

[1] Anschuetz, R., Scherwood, H.: When is a function's inverse equal to its reciprocal?. College Math. J. 27, 388-393 (1996)

[2] Boros, Z.: Talk Given During the Fifty International Symposium on Functional Equations. Hajdúszoboszló, Hungary, 17-24 June (2012)

[3] Brillouët-Belluot, N.: Problem posed during The Forty-ninth International Symposium on Functional Equations. Aequat. Math. 84, 312 (2012)

[4] Chen, L., Shi, Y.: The real solutions of functional equation $f^{[m]}=1 / f$. J. Math. Res. Exposition 28, 323-330 (2008)

[5] Cheng, R., Dasgupta, A., Ebanks, B.R., Kinch, L.F., Larson, L.M., McFadden, R.B.: When does $f^{-1}=1 / f$ ?. Amer. Math. Monthly 105, 704-716 (1998)

[6] Daepp, U., Gorkin, P.: Reading, Writing, and Proving: A Closer Look at Mathematics. Undergraduate Texts in Mathematics. Springer, New York (2003)

[7] Euler, R., Foran, J.: On functions whose inverse is their reciprocal. Math. Mag. 54, 185189 (1981)

[8] Jarczyk, W., Morawiec, J.: Note on an equation occurring in a problem of Nicole Brillouët-Belluot. Aequat. Math. 84, 227-233 (2012)

[9] Kuczma, M.: Functional equations in a single variable. Monografie Matematyczne, vol. 46. PWN, Polish Scientific Publishers, Warsaw (1968)

[10] Kuczma, M., Choczewski, B., Ger, R.: Iterative functional equations. Encyclopedia of mathematics and its applications, vol. 32. Cambridge University Press, Cambridge (1990)

[11] Massera, J.L., Petracca, A.: Sobre la ecuación funcional $f(f(x))=1 / x$. Rev. Un. Mat. Argentina. 11, 206-211 (1946)

[12] Morawiec, J.: On a problem of Nicole Brillouët-Belluot. Aequat. Math. 84, 219225 (2012)

[13] Ng, C.T., Zhang, W.: When does an iterate equal a power?. Publ. Math. Debrecen 67, 79-91 (2005)

Janusz Morawiec

Institute of Mathematics

University of Silesia

Bankowa 14

PL-40-007 Katowice

Poland

e-mail: morawiec@math.us.edu.pl

Received: March 7, 2013

Revised: April 13, 2013 